\title{
Druckverhältnisse in großen Kaplan- Turbinen und deren Auswirkungen auf die Überlebensraten von Fischen
}

Druckunterschiede werden als eine der wesentlichen Schädigungsursachen von Fischen beim Passieren von Turbinen diskutiert. Bisherige Untersuchungen zeigen allerdings nur für wenige Arten und Lebensstadien hohe Sensibilitäten hinsichtlich Barotrauma. Wenn man davon ausgeht, dass die meisten abwandernden Fische oberflächennah wandern und unter der Berücksichtigung der sehr geringen potenziellen Schädigungsraten durch Barotrauma in großen Kaplan-Turbinen sind Auswirkungen auf potamodrome Fischpopulationen wahrscheinlich ohne Bedeutung bzw. nicht nachweisbar.

Walter Reckendorfer

\section{Einleitung}

Durch den Kraftwerksbetrieb können Fische, welche die Turbine passieren, geschädigt werden. Neben dem Kontakt mit einer Turbinenschaufel werden Druckunterschiede, wie sie beim Passieren einer Turbine auftreten, als eine der wesentlichen Schädigungsursachen diskutiert.

Studien in den letzten Jahren haben gezeigt, dass die Schädigung durch Barotrauma durch das Verhältnis zwischen dem Druck in der Akklimatisationstiefe (PA) und dem niedrigsten Druck während der Turbinenpassage (PE) bestimmt wird, wobei der Tiefstwert in der Literatur auch als Nadir bezeichnet wird. Hohe Drücke spielen als Schädigungsmechanismus in der Regel keine Rolle [1]. Verschiedene Fischarten und Entwicklungsstadien reagieren dabei sehr unterschiedlich hinsichtlich Änderungen im Druck.

\section{Druckverhältnisse in großen Kaplan-Turbinen}

Bild 1 zeigt die niedrigsten Drücke, welche an fünf großen Kaplan-Turbinen gemessen wurden. Die Fallhöhe lag zwischen

\section{Kompakt}

- Die Schädigung durch Barotrauma ist art- und stadienspezifisch.

- Bei Druckverhältnissen, wie sie in großen KaplanTurbinen auftreten, kommt es bei Eiern und Larven in der Regel zu keinen druckbedingten Mortalitäten, weisen Juvenile und Adulte vieler untersuchter Arten eine hohe Widerstandfähigkeit auf und wurden bisher nur für wenige Arten signifikante Mortalitäten festgestellt.

- Bei großen Kaplan-Turbinen dürften daher druckbedingte Mortalitätsraten nur eine geringe Rolle spielen. ca. $15 \mathrm{~m}$ und $35 \mathrm{~m}$, der Turbinendurchfluss zwischen $230 \mathrm{~m}^{3} / \mathrm{s}$ und $576 \mathrm{~m}^{3} / \mathrm{s}$.

Die mittleren Nadirwerte schwankten für die einzelnen Versuche zwischen $84 \mathrm{kPa}$ und $194 \mathrm{kPa}$ (Mittelwert = $134 \mathrm{kPa}$ ). Je höher der Durchfluss ist, desto geringere Drücke treten in den Turbinen auf, d. h. desto größer ist die Wahrscheinlichkeit einer druckbedingten Schädigung.

Der Median über alle Untersuchungen lag bei $135 \mathrm{kPa}$, also über dem normalen atmosphärischen Druck von $101 \mathrm{kPa}$ (Luftdruck). Nur bei ca. $15 \%$ der Turbinenpassagen treten überhaupt Nadirwerte unter dem atmosphärischen Druck auf, die theoretisch zu Schädigungen führen können. Bei nur ca. 1 \% der Turbinenpassagen traten Drücke von unter $50 \mathrm{kPa}$ auf (Tabelle 1).

\section{Mortalitäten durch Barotrauma}

\subsection{Eier}

Bei Fischeiern traten bisher bei keiner Untersuchung druckbedingte Schädigungen auf. Eier vom Weißen Stör (Acipenser trans-

Tabelle 1: Quantile der Nadir-Werte aus den Untersuchungen mit Sensorfischen (Quelle: Reckendorfer, Daten aus Bild 1)

\begin{tabular}{|c|c|}
\hline Quantile [\%] & Nadir [kPa] \\
\hline 1 & 46 \\
\hline 5 & 75 \\
\hline 10 & 91 \\
\hline 15 & 101 \\
\hline 25 & 115 \\
\hline 75 & 135 \\
\hline 90 & 158 \\
\hline 95 & 175 \\
\hline 99 & 185 \\
\hline
\end{tabular}




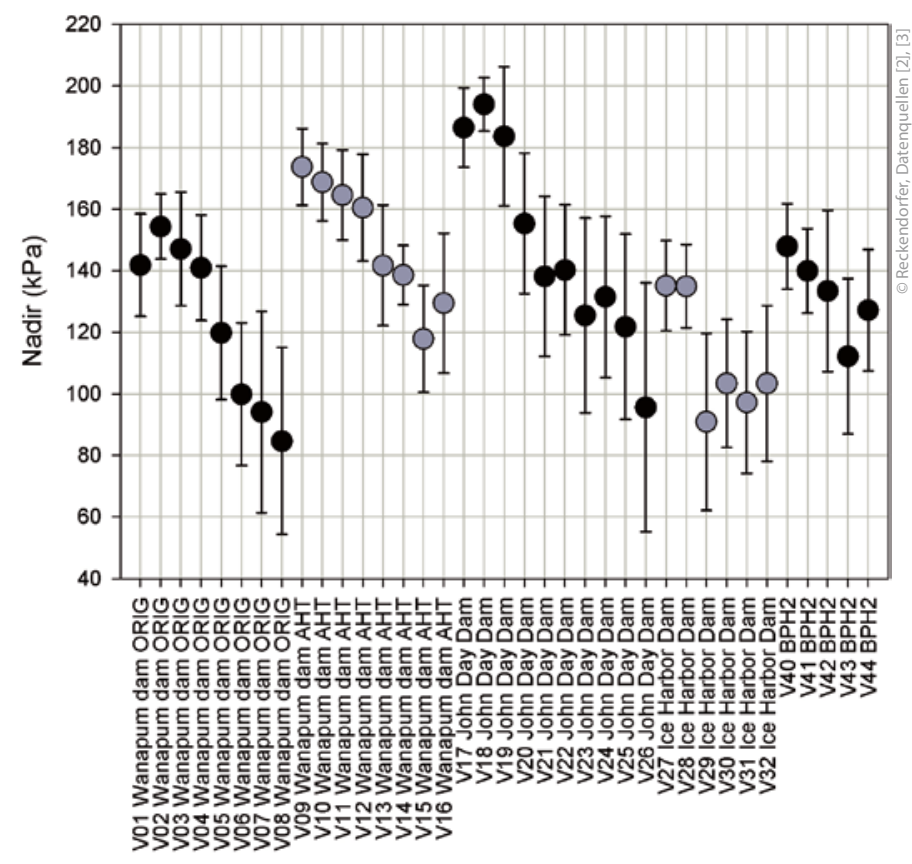

Versuch

montanus), vom Australischen Silberbarsch (Bidyanus bidyanus), und vom Australischen Goldbarsch (Macquaria ambigua) zeigten selbst bei extrem niedrigen Drücken (Nadir von $1 \mathrm{kPa}$ bis $5 \mathrm{kPa}$ ), wie sie nur in Extremfällen beim Turbinenbetrieb auftreten können, kein Anzeichen von Schädigung (Tabelle 2).

\subsection{Larven}

Bei Fischlarven trat beim Großteil der untersuchten Arten keine druckbedingte Mortalität auf. Larven des Weißen Störs (Acipenser transmontanus) zeigten am neunten Tag mit Beginn des Fressens eine erhöhte Empfindlichkeit mit Mortalitätsraten von $10 \%$ bis $20 \%$, alle jüngeren und älteren Larven überlebten selbst Nadirwerte von $5 \mathrm{kPa}$ bzw. Druckreduktionen auf 1/20 des Akklimatisationsdrucks (Tabelle 3). Larven des Silberbarsches
Bild 1: Nadir-Werte (MW + SD) für verschiedene Turbinen (BPH2 - Bonneville Dam, Powerhouse 2) und Versuche (V01 bis V44); für die jeweilige Turbine sind die Versuche von niedrigem zu hohem Durchfluss geordnet

(Bidyanus bidyanus), des Goldbarsches und des Murray-Dorsches (Maccullochella peelii) überlebten Druckänderungen auf $1 / 9$ des Akklimatisationsdrucks $($ Nadir $=11 \mathrm{kPa})$.

\subsection{Juvenile und Adulte}

Einige Arten (Aale, Neunaugen, Störe) sind gegenüber niedrigen Drücken bzw. Druckänderungen unempfindlich. Aale und Störe überlebten selbst Nadir-Werte von ca. 2 kPa bzw. eine Druckreduktionen auf 1/50 des Akklimatisationsdrucks (Tabelle 4).

Für einige Arten konnte die Mortalitätsrate durch logistische Regressionen in Abhängigkeit von PA/PE dargestellt werden:

$$
P_{\text {mort }}=\left(\frac{\left(E X P\left(\beta_{0}+\beta_{1} \ln (P A / P E)\right)\right.}{\left(1+E X P\left(\beta_{0}+\beta_{1} \ln (P A / P E)\right)\right.}\right)
$$

Tabelle 2: Überlebensraten von Eiern bei unterschiedlichen Nadir-Werten (Quelle: [4], [5])

\begin{tabular}{|c|c|c|c|c|}
\hline Art & PA [kPa] & PE, Nadir [kPa] & \multicolumn{1}{c|}{ PA/PE [-] } & Überlebensrate [\%] \\
\hline A. transmontanus & 101 & $5-39$ & $20,2-2,6$ & 100 \\
\hline B. bidyanus & 101 & $11-101$ & $9,2-1,0$ & 100 \\
\hline M. ambigua & 101 & $11-101$ & $9,2-1,0$ & 100 \\
\hline
\end{tabular}

Tabelle 3: Überlebensraten von Larven bei unterschiedlichen Nadir-Werten (dph: days post hatching) (Quelle: [4], [5])

\begin{tabular}{|c|c|c|c|c|c|c|}
\hline Art & Alter, Größe & $\mathrm{PA}[\mathrm{kPa}]$ & $\mathrm{PE}, \mathrm{Nadir}[\mathrm{kPa}]$ & PA/PE [-] & Überlebensrate [\%] & Quelle \\
\hline \multirow[t]{3}{*}{ A. transmontanus } & $0-8 \mathrm{dph}$ & 101 & $5-39$ & $20,2-2,6$ & 100 & [4] \\
\hline & $9 \mathrm{dph}$ & 101 & $18-39$ & $5,5-2,6$ & $80-90$ & [4] \\
\hline & $10-75 \mathrm{dph}$ & 101 & $5-39$ & $20,2-2,6$ & 100 & [4] \\
\hline B. bidyanus & $10-22 \mathrm{dph}$ & 101 & $11-101$ & $9,2-1,0$ & 100 & [5] \\
\hline M. ambigua & 10-18 dph & 101 & $11-101$ & $9,2-1,0$ & 100 & {$[5]$} \\
\hline M. peelii & $3-25 \mathrm{dph}$ & 101 & $11-101$ & $9,2-1,0$ & 100 & [5] \\
\hline
\end{tabular}


Tabelle 4: Überlebensraten von juvenilen und adulten Fischen bei unterschiedlichen Nadir-Werten (ÜR: Überlebensrate) (Quelle: [6], [7], [8])

\begin{tabular}{|c|c|c|c|c|c|c|c|}
\hline & Art & Größe $[\mathrm{mm}]$ & PA [kPa] & PE, Nadir $[\mathrm{kPa}]$ & PA/PE [-] & ÜR [\%] & Quelle \\
\hline Amerikanischer Aal & Anguilla rostrata (Silberaal) & $216-686$ & 101 & $1,6-8,4$ & $12,0-63,1$ & 100 & {$[6]$} \\
\hline Amerikanischer Aal & Anguilla rostrata (Gelbaal) & $230-423$ & 101 & $1,2-8,1$ & $12,5-84,2$ & 100 & {$[6]$} \\
\hline White Sturgeon & Acipenser transmontanus $n$ & $145-338$ & 146,2 & $1,8-10,3$ & $9,8-56,1$ & 100 & {$[7]$} \\
\hline Pazifisches Neunauge & Entosphenus tridentatus & $127-183$ & 101 & 14 & 10,6 & 100 & {$[8]$} \\
\hline Westliches Bachneunauge & Lampetra richardsonii & $80-124$ & 101 & 14 & 10,6 & 100 & {$[8]$} \\
\hline
\end{tabular}

Tabelle 5: Koeffizienten der logistischen Regression für bisher untersuchte Arten (Quelle: [7], [9], [10])

\begin{tabular}{|c|c|c|c|c|c|c|c|}
\hline Name & Art & Typ & Größe [mm] & $\beta 0[-]$ & $\beta 1[-]$ & Quelle \\
\hline Königslachs & Oncorhynchus tshawytscha & physostom & & $-5,56$ & 3,85 & {$[9]$} \\
\hline Australian bass & Macquaria novemaculeata & physoclist & $73-26$ & $-5,72$ & 2,68 & {$[10]$} \\
\hline Gudgeon & Hypseleotris spp. & physostom & $23-50$ & $-5,70$ & 1,99 & {$[10]$} \\
\hline Murray-Dorsch & M.peelii & physostom & $54-80$ & $-7,33$ & 2,79 & {$[10]$} \\
\hline Silver perch & Bidyanus bidyanus & physoclist & $45-112$ & $-3,91$ & 1,39 & {$[10]$} \\
\hline Sander vitreum & Sander vitreum & physoclist & $178-319$ & $-4,96$ & 2,84 & {$[7]$} \\
\hline Muskellunge & Esox masquinongyX Esox lucius) & physostom & $122-259$ & $-3,93$ & 1,96 & {$[7]$} \\
\hline
\end{tabular}

In Tabelle 5 sind die Regressionskoeffizienten für diese Arten angeführt, eine grafische Darstellung des Zusammenhanges zeigt Bild 2.

Der Unterschied zwischen physoclisten Arten und physostomen Arten ist nicht so ausgeprägt wie erwartet. Die Unterschiede sind zum Teil auch Methoden bedingt, da sowohl unterschiedliche Endpunkte dokumentiert wurden als auch andere Versuchsbedingungen vorlagen: Von Brown et al. [9] und Pflugrath et al. [10] wurden als Endpunkt nicht die Überlebensraten dokumentiert, sondern artspezifische Parameter, die mit der Überlebensrate bzw. Mortalitätsrate signifikant korreliert waren, es wurde also nicht die tatsächliche Mortalität ermittelt, sondern ein Index („mortally injured“) [11]. Diese
Vorgehensweise kann die tatsächlichen Überlebensrate beträchtlich unterschätzten, da das Auftreten jeder einzelnen Verletzung, die einen signifikanten Zusammenhang mit der Mortalität zeigt, dazu führt, das ein Fisch als „mortally injured“ bzw. „tot“ klassifiziert wird. Von Brown et al. [7] wurden die Parameter, welche für den Königslachs entwickelt wurden [6], übernommen, d. h. keine artspezifischen Parameter entwickelt. Die Autoren nehmen an, dass dadurch die Mortalitätsraten („mortally injured“) für Muskellunge und Zander im Vergleich zum Königslachs überschätzt wurden. Unterschiedliche Versuchsbedingungen betreffen die Gassättigung. Die Versuche am Königlachs [9] wurden bei Gasübersättigung (113\% bis $128 \%$ ) durchgeführt (Bild 3). Bei diesen Konzent-

Tabelle 6: Mortalitätsindex („,mortally injured“) verschiedener Fischarten (adulte und juvenile, oberflächenadaptiert) bei unterschiedlichen Nadir-Werten (Quantile der beobachteten Nadir-Werte); Berechnungen anhand der Formeln in Tabelle 5 (Quelle: Reckendorfer, Daten: [7], [9], [10])

\begin{tabular}{|c|c|c|c|c|c|c|c|c|c|c|c|}
\hline & \multicolumn{10}{|c|}{ Mortalitätsindex } & $\begin{array}{c}\text { Kumulierte Mortalität } \\
\text { (Nadir }<101 \mathrm{kPa})\end{array}$ \\
\hline Quantile [\%] & 1 & 5 & 10 & 15 & 25 & 50 & 75 & 90 & 95 & 99 & - \\
\hline Nadir [kPa] & 46 & 75 & 91 & 101 & 115 & 135 & 158 & 175 & 185 & 203 & $0,17 \%$ \\
\hline Königslachs (112-127 \% TDG) & 7,1 & 1,2 & 0,6 & 0,4 & 0,2 & 0,1 & 0,1 & 0,0 & 0,0 & 0,0 & 0,0 \\
\hline Australian bass & 2,6 & 0,7 & 0,4 & 0,3 & 0,2 & 0,2 & 0,1 & 0,1 & 0,1 & 0,1 & $0,09 \%$ \\
\hline Gudgeon & 1,5 & 0,6 & 0,4 & 0,3 & 0,3 & 0,2 & 0,1 & 0,1 & 0,1 & 0,1 & $0,07 \%$ \\
\hline Murray-Dorsch & 0,6 & 0,2 & 0,1 & 0,1 & 0,0 & 0,0 & 0,0 & 0,0 & 0,0 & 0,0 & $0,02 \%$ \\
\hline Silver perch & 5,6 & 2,9 & 2,3 & 2,0 & 1,6 & 1,3 & 1,1 & 0,9 & 0,9 & 0,8 & $0,39 \%$ \\
\hline Sander vitreum & 6,0 & 1,6 & 0,9 & 0,7 & 0,5 & 0,3 & 0,2 & 0,1 & 0,1 & 0,1 & $0,20 \%$ \\
\hline Muskellunge & 8,3 & 3,4 & 2,4 & 2,0 & 1,5 & 1,1 & 0,8 & 0,7 & 0,6 & 0,5 & $0,44 \%$ \\
\hline
\end{tabular}




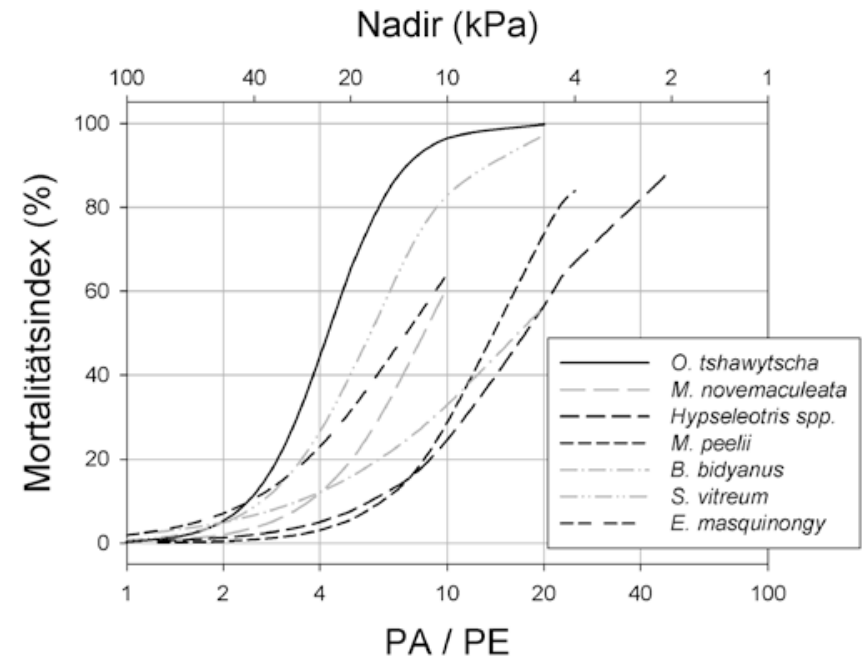

Bild 2: Mortalität (Index) verschiedener Fischarten (oberflächenadaptiert) in Abhängigkeit vom Nadir (grau: physoclist, schwarz: physostom)

rationen reagieren die Fische wesentlich empfindlicher auf Druckänderungen als bei normaler Gassättigung ([9], [12]).

Für Oberflächen-adaptierte Fische ergeben sich im Großteil der untersuchten Fälle keine Mortalitäten, da nur bei ca. $15 \%$ der Turbinenpassagen überhaupt Nadir-Werte unter dem nor-

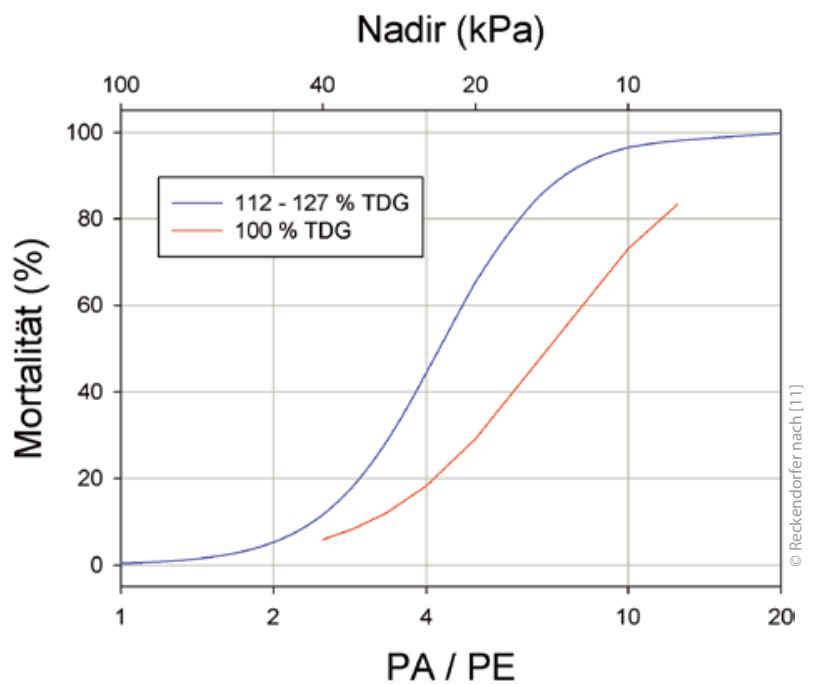

Bild 3: Mortalität (Index) des Königlachses (O. tshawytscha, oberflächenadaptiert) bei unterschiedlichen Gaskonzentrationen (TDG: total dissolved gas) im Wasser

malen atmosphärische Druck auftraten, die theoretisch zu Schädigungen führen können (Tabelle 6). Die in der Tabelle 6 ausgewiesenen Mortalitäten geben zwar auch oberhalb des atmosphärischen Luftdrucks Mortalitäten an, diese ergeben sich aber Formel bedingt, da die logistischen Regres-

\section{WASSERWIRTSCHAFT}

Das Fachmagazin für Wasser und Umwelt.

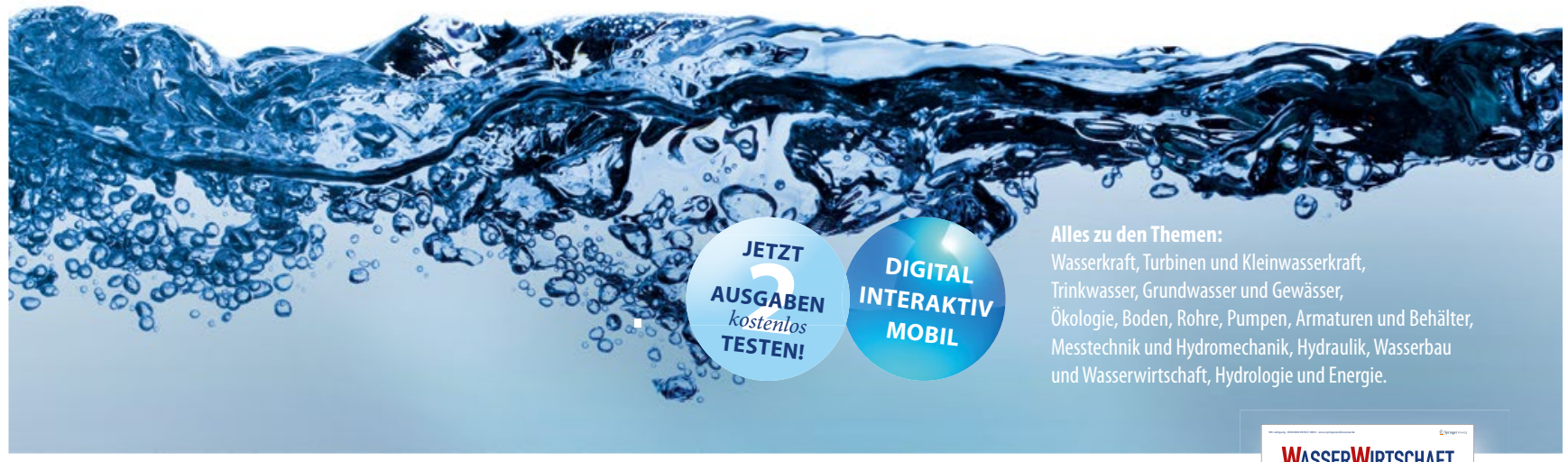

Fundierte Berichte aus Forschung und Wissenschaft - WasserWirtschaft bietet hohe technologische Kompetenz durch praxisnahe Fachbeiträge. Nutzen Sie den Vorteil der zehn Printausgaben im Jahr zum Vorzugspreis und exklusiv dem interaktiven e-magazin mit der beeindruckenden Wissensdatenbank des Onlinearchivs mit pdf-Download.

www.meinfachwissen.de/wawi

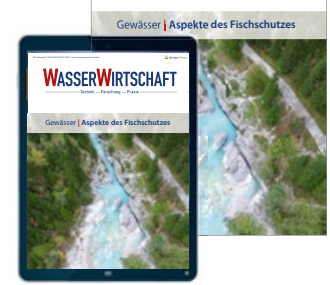


sionen nicht durch den Ursprung laufen. Bereiche mit niedrigen Drücken, die für Fische letal sein können, sind örtlich eng begrenzt, bei nur ca. $1 \%$ der Turbinenpassagen traten Drücke von unter $50 \mathrm{kPa}$ auf. Integriert man Auftrittshäufigkeiten und Mortalitäten über den relevanten Bereich $(<101 \mathrm{kPa})$, so ergeben sich bei allen bisher untersuchten Arten für oberflächenadaptierte Fische Gesamtmortalitäten von deutlich unter einem Prozent. Wenn man davon ausgeht, dass die Turbinen in der Regel im Bereich der höchsten Effizienz betrieben werden, wären die Mortalitätsraten zwar etwas höher, aber immer noch sehr gering.

Da der Großteil der abwärtswandernden potamodromen Fische Larven und Juvenile sind, die in der Regel oberflächenadaptiert sind, sind Auswirkungen auf potamodrome Fischpopulationen durch Turbinen bedingtes Barotrauma an großen Anlagen wahrscheinlich nicht signifikant bzw. nicht nachweisbar. Einschränkend muss allerdings hinzugefügt werden, dass bisher keine vergleichbaren Untersuchungen an Cypriniden durchgeführt wurden, die den Hauptteil der Fische in großen europäischen Gewässern darstellen. Um auch für diese Arten belastbare Daten zu erhalten, wird derzeit ein Forschungsprojekt von Oesterreichs Energie Forschung \& Innovation „Flussabwärts gerichtete Fischwanderung an mittelgroßen Fließgewässern in Österreich: Populationsbiologische Grundlagen und Implikationen für den Fischschutz und Fischabstieg" durchgeführt, welches von der Österreichischen Forschungsförderungsgesellschaft (FFG) im Rahmen eines Collective Research Projektes (857 801) gefördert wird [13].

\section{Autor}

\section{Mag. Dr. Walter Reckendorfer}

Verbund Hydro Power GmbH

Europaplatz 2

1150 Wien, Österreich

walter.reckendorfer@verbund.com

\section{Literatur}

[1] Cada, G. F.: A review of studies relating to the effects of propeller-type turbine passage on fish early life stages. In: North American Journal of Fisheries Management 10 (1990), Nr. 4, S. 418-426.

[2] Carlson, T. J.; Duncan, J. P.; Deng, Z.: Data Overview for Sensor Fish Samples Acquired at Ice Harbor, John Day, and Bonneville II Dams in 2005, 2006, and 2007. Report prepared for the U. S. Army Corps of Engineers, 2008.

[3] Dauble, D. D.; Deng, Z.; Richmond, M. C. et al.: Biological Assessment of the Advanced Turbine Design at Wanapum Dam, 2005. Report prepared for the U.S. Department of Energy Office of Energy Efficiency and Renewable Energy Wind and Hydropower Technologies, 2007.

[4] Brown, R. S.; Cook, K. V.; Pflugrath, B. D. et al.: Vulnerability of larval and juvenile white sturgeon to barotrauma: can they handle the pressure? In: Conservation Physiology 1 (2013), Nr. 1.

[5] Boys, C. A.; Robinson, W.; Miller, B. et al.: How low can they go when going with the flow? Tolerance of egg and larval fishes to rapid decompression. In: Biology Open 5 (2016), Nr. 6, S. 786-793.

[6] Pflugrath, B. D.; Harnish, R.; Rhode, B. et al.: American eel state of buoyancy and barotrauma susceptibility associated with hydroturbine passage. In: Knowledge \& Management of Aquatic Ecosystems 420 (2019), Nr. 20.

[7] Brown, R. S.; Walker, R. W.; \& Stephenson, J. R.: A Preliminary Assessment of Barotrauma Injuries and Acclimation Studies for Three Fish Species (No. PNNL-24720). Pacific Northwest National Lab. (PNNL), Richland, WA (United States), 2015.

[8] Colotelo, A. H.; Pflugrath, B. D.; Brown, R. S. et al.: The effect of rapid and sustained decompression on barotrauma in juvenile brook lamprey and Pacific lamprey: implications for passage at hydroelectric facilities. In: Fisheries Research 129 (2012), S. 17-20.

[9] Brown, R. S.; Carlson, T. J.; Gingerich, A. J. et al.: Quantifying mortal injury of juvenile Chinook salmon exposed to simulated hydro-turbine passage. In: Transactions of the American Fisheries Society 141 (2012), Nr. 1, S. 147-157.

[10] Pflugrath, B. D.; Boys, C. A.; Cathers, B.: Predicting hydraulic structure-induced barotrauma in Australian fish species. In: Marine and Freshwater Research 69 (2018), Nr. 12, S. 1 954-1 961.

[11] McKinstry, C. A.; Carlson, T. J.; Brown, R. S.: Derivation of mortal injury metric for studies of rapid decompression of depth-acclimated physostomous fish (No. PNNL-17080). Pacific Northwest National Lab. (PNNL), Richland, WA (United States), 2007.

[12] Colotelo, A.; Brown, R.; Carlson, T. et al.: Injury mechanisms for juvenile Chinook salmon passing through hydropower turbines.

[13] Schneider, J.; Ratschan, C.; Heisey, P. et al.: Flussabwärts gerichtete Fischwanderung an mittelgroßen Fließgewässern in Österreich. In: WasserWirtschaft 107 (2017), Heft 12, S. 33-38.

\section{Walter Reckendorfer}

Pressure conditions in large Kaplan turbines and their impact on the survival rates of fish

Barotrauma is discussed as one of the major causes of damage to fish as they pass turbines. However, previous studies show high sensitivities to barotrauma only for a few species and life stages. Assuming that downstream migrating larvae and juveniles are surface orientated and taking into account the very low rates of barotrauma damage, the impact on potamodromous fish populations is in a range that is undetectable under natural conditions.

\section{SpringerProfessional.de}

Turbinenbedingte Schädigungen

Reckendorfer, W.; Loy, G.; Ulrich, J.; et al.: Maßnahmen zum Schutz der Fischpopulation - die Sicht der Betreiber großer Wasserkraftanlagen. In: WasserWirtschaft, Ausgabe 2-3/2017.

Wiesbaden: Springer Vieweg, 2017.

www.springerprofessional.de/link/12114410

Reckendorfer, W.; Schmalfuß, R.; Frik, G.; et al.: Ökologische Maßnahmen zum Schutz der Fischpopulation. In: WasserWirtschaft, Ausgabe 2-3/2018. Wiesbaden: Springer Vieweg, 2018. www.springerprofessional.de/link/15499166 